\title{
The impact of body mass index and Western advertising and media on eating style, body image and nutrition transition among Jordanian women
}

\author{
Hala N Madanat ${ }^{1,2, *}$, Ralph B Brown ${ }^{3}$ and Steven R Hawks ${ }^{2}$ \\ 'Department of Community Health, School of Science and Health, Utah Valley State College, 800 W University \\ Parkway, Orem, UT 84058, USA: ${ }^{2}$ Department of Health Science, College of Health and Human Performance, \\ Brigham Young University, Provo, UT, USA: ${ }^{3}$ Department of Sociology, College of Family, Home, and Social \\ Sciences, Brigham Young University, Provo, UT, USA
}

Submitted 9 June 2006: Accepted 29 November 2006: First published online 22 February 2007

\begin{abstract}
Objectives: To identify the impact of body mass index (BMI) and Western advertising and media on the stage of the nutrition transition among Jordanian women, and to evaluate their impact on eating styles and body image.

Design: A randomised cross-sectional survey that included a variety of culturally measured Likert-type scales and body size images. In addition, BMI was calculated based on measured height and weight.

Setting: In the homes of the participants. The data were collected by female interviewers who worked for the Jordan Department of Statistics.

Subjects: The sample was based on a random and representative selection of 800 mostly urban Jordanian women. A pre-test sample of 100 women was also used to validate the instruments.

Results: Women tended to agree that they ate based on emotional cues. They had high levels of disordered eating attitudes and behaviours and $42.1 \%$ were considered restrained eaters. However, these women also had higher than expected body esteem levels and desired a healthy body size. As expected, being obese was associated with a desire to lose weight, being a restrained and emotional eater, and having more disordered eating attitudes and behaviours. Similarly, Western advertising and media were associated with restrained and emotional eating, desired weight loss, and disordered eating attitudes and behaviours.

Conclusions: There is a need to develop health education materials that explain the influence of obesity on health and the negative psychological and physical consequences of restrained and emotional eating, building on the current cultural preferences of healthy body size. Further implications and suggestions for future research are discussed.
\end{abstract}

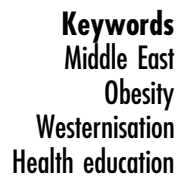

The nutrition transition is a well-documented global phenomenon characterised by the adoption of modern diets high in fat, sugar and salt, and the abandonment of traditional diets high in fibre, grains, fruits and vegetables ${ }^{1}$. This transition is accompanied by an increase in the rates of both obesity and chronic diseases including diabetes, hypertension and stroke, and it is also used as a predictor of high obesity levels in a country ${ }^{2,3}$.

\section{The nutrition transition model}

In the present study, the model of the nutrition transition proposed by Hawks et $a l .{ }^{4}$ is used as the theoretical framework. This model suggests that, prior to the nutrition transition, people are physical eaters; they generally do not overeat and their food intake is based on what appeals to them and what satisfies their hunger. The model further predicts that due to economic and demographic transitions there will be a shift in diet composition as well as a shift in eating styles. Initially, individuals shift from physical eating to external eating, which is associated with increased obesity levels. The term 'external eating' refers to eating based on social and environmental cues; such as being at a party or out with friends, or eating triggered by something in the immediate surroundings such as seeing a restaurant or smelling food ${ }^{4}$. During this stage, and due in part to Western media, there is an increase in preference for body thinness versus plumpness, which often leads to restrictive dieting and the denial of hunger and may eventually contribute to emotional eating as a response to feelings of deprivation. 
'Emotional eating' refers to eating in the absence of hunger due to emotional triggers such as boredom, anger and frustration. Thus, the replacement of social and environmental eating styles, by restrictive dieting and emotional eating, leads to both negative physical and psychological consequences. These new eating styles are often associated with overeating and increased rates of nutrition-related non-communicable diseases (NR-NCDs) such as diabetes and stroke ${ }^{4}$.

\section{The nutrition transition in Jordan}

Jordan has been moving rapidly through the nutrition transition. Levels of overweight and obesity are on the rise and reaching epidemic proportions ${ }^{5}$. Jordan also faces a unique situation in terms of its demographic transition. In 1980 the population was 2.2 million; by 1999 it had doubled; and it is expected to double again by 2035 . The current median age is 19.3 years, reflecting a young population ${ }^{5,6}$. These demographic changes are partly due to health-care reforms that have resulted in decreased infant mortality rates (from 160/ 1000 live births in 1950 to 20/1000 live births in 2001), decreased fertility rates (from 7.8 in 1970 to 3.6 in 2003) and increased life expectancy (from 54 in 1970 to 72 years today) ${ }^{6}$.

These changes have been accompanied by rapidly increasing urbanisation. Between 1965 and 2000 the urban population increased from 46 to $75 \%$, which paralleled changes in the nutrition transition such as reduced famines and increased NR-NCDs ${ }^{6}$. It is estimated that between 1965 and 1992 the per capita daily energy intake increased from 2165 to $3459 \mathrm{kcal}^{5}$. In $1964,70.7 \%$ of dietary energy supply was from carbohydrates, $10.6 \%$ from proteins and $18.7 \%$ from fat. In 2000, the percentages were 64.0, 10.8 and 25.2, respectively ${ }^{5}$.

Data on the nutritional status of adults in Jordan are limited. A 2003 study reported the prevalence of overweight persons as 32\% and the prevalence of obesity as $13 \%{ }^{7}$. The 2002 Demographic and Health Survey ${ }^{8}$ found that the majority of women (53.9\%) were overweight or obese and $41.1 \%$ fell in the normal range of body mass index (BMI) of $18.5-24.9 \mathrm{~kg} \mathrm{~m}^{-2}$.

Data on risk factors associated with NR-NCDs indicate a high prevalence of these risk factors in this population. Hypertension affects $22.2 \%$ of the population. Diabetes affects $6.4 \%$ of the population, while the percentage of individuals with high blood cholesterol is $20.9^{7}$. Thus, based on this information, two hypotheses are formed about the nutrition transition among Jordanian women.

1. The nutrition transition is underway in Jordan. Specifically, most Jordanian women will be restrained eaters.

2. Western advertising and media influences will explain the eating style and body size preferences of Jordanian women.

\section{Methods}

\section{Study design and sample}

After receiving approval from the Institutional Review Board at Brigham Young University, women from all across Jordan were selected at random to participate in the study. The sample for this study was based on the frame provided by the 2004 Jordanian Population and Housing Census. The frame consists of data on each primary sampling unit (PSU), each of which is composed of 100 housing units. It excluded the population living in remote areas, as well as those living in collective dwellings such as hotels, hospitals and the like.

The sample was selected using a stratified three-stage cluster sampling design. For data stratification, the population localities in each of the 12 governorates were divided into two strata: urban and rural. For this survey, each locality with 5000 persons or more as revealed by the 2004 Population and Housing Census was considered 'urban'. The remaining localities were considered 'rural'.

In the first stage of sampling 80 PSUs were selected. These units were distributed across governorates according to each unit's weight in terms of the number of households it contains. The PSUs were selected using probability proportionate to size with a systematic selection procedure. In the second stage, the equal secondary sampling units were selected (10 households per PSU). In the third stage, one eligible female was selected.

This study utilised a cross-sectional survey design, with women from a variety of religious, socio-economic and educational backgrounds being the target population. The sample included 800 women aged 18 years and over. Professional interviewers from the Department of Statistics met with the women in their homes. The response rate was 99.6\%. Pregnant women were also excluded since BMI calculations would be affected. It is noteworthy that the interviewers were all females to ensure cultural sensitivity. The data collection was done in January 2006. The data were entered, checked and rechecked for accuracy.

\section{Instruments}

No single scale currently measures Hawks et al.'s nutrition transition model comprehensively. Thus several scales were used in this study. All of these scales have been previously validated in the literature. However, a pre-test was carried out to validate them in this population. In addition, a consent form was included with the instrument which explained the purpose of the study, that participation was voluntary, that the participant may withdraw at any point, and that no identifying information would be collected.

\section{The Motivation for Eating Scale (MFES)}

The MFES is a Likert-type scale with 43 items that measure the participant's degree of agreement with various statements related to motivations for eating. It has four subscales: intuitive eating, emotional eating, social eating 
and environmental eating. Thus this survey allowed the measurement of the different eating styles except for restrained eating?

\section{Eating Habits Questionnaire (RS)}

The Eating Habits Questionnaire, also known as the Revised Restraint Scale (RS), is a 10-item scale that measures restrictive dieting. The cut-off point is based on the median. Thus, individuals scoring at the median or higher are considered restrained eaters ${ }^{10,11}$.

\section{Eating Attitudes Test (EAT-26)}

This scale was used to measure disordered eating attitudes and behaviours. It is a Likert-type scale with 26 items and has been translated to Arabic ${ }^{12-14}$. Scoring 20 or more on this scale indicates that an individual may have disordered eating attitudes and behaviours.

\section{Sociocultural Attitudes Towards Appearance Scale} (SATAQ-3)

SATAQ-3 was used to assess the impact of societal and media influences on body image and eating disturbances $^{15}$. It is a 30-item scale including four subscales: information, pressures, internalisation-general and internalisation-athlete, that measure the societal and media impact on body image.

\section{Body silbouettes}

Body size preferences were measured using Stunkard et al.'s silhouettes ${ }^{16}$. Participants were asked four questions: (1) Which figure represents your current size? (2) Which figure represents your ideal size? (3) Which figure best represents a healthy figure? (4) Which figure would be preferred by the opposite gender?

\section{Body Esteem Scale (BES)}

BES is a 23-item Likert-type scale with three subscales: $\mathrm{BE}$-appearance, BE-weight and BE-attribution. These subscales assess, respectively, general feelings about appearance, weight satisfactions and evaluations attributed to others about one's body and appearance ${ }^{17}$.

\section{Demographics}

Subjects' demographics - including age, area of residence, socio-economic status measured by family's annual income and socio-economic index based on the ownership of several devices - were also collected and controlled for in the analyses. Individuals having up to eight of the devices were considered of low socio-economic status, those with nine to 15 of medium socio-economic status, and those with 16 or more of those devices were considered of high socio-economic status. These cut-off points were used based on the recommendations of the Department of Statistics, which routinely uses this question to categorise the socio-economic status of Jordanian families.

\section{$B M I$}

Another variable of interest was BMI. Interviewers were asked to collect height and weight from the surveyed women using standardised equipment and methodology, which were used to calculate BMI. Four different categories of BMI were used: underweight $(\mathrm{BMI}<18.5$ $\mathrm{kg} \mathrm{m}^{-2}$ ), normal $\left(18.5 \mathrm{~kg} \mathrm{~m}^{-2}<\mathrm{BMI}<24.9 \mathrm{~kg} \mathrm{~m}^{-2}\right)$, overweight $\left(24.9 \mathrm{~kg} \mathrm{~m}^{-2}<\mathrm{BMI}<29.9 \mathrm{~kg} \mathrm{~m}^{-2}\right)$ and obese (BMI $>29.9 \mathrm{~kg} \mathrm{~m}^{-2}$ ).

\section{Validity and reliability}

For this research a native speaker translated all of these instruments to Arabic, and then they were independently back-translated to English by another native speaker to guarantee accurate translation. Face validity was assessed using a focus group from the target population to ensure understanding of the questions and their cultural appropriateness. The focus group revealed that item 20 on the BES, 'My looks help me to get dates', was culturally inappropriate and was thus substituted with 'My looks help me get attention from males'.

A pre-test group of 100 women was also used to assess both validity and reliability of these instruments in Jordan. Factor analyses were carried out to identify which items loaded appropriately in each of the subscales. Alpha coefficients were computed to measure internal consistency. Reliability analyses were done for each of the subscales or scales used in the instrument using Cronbach's $\alpha$. The factor analyses used were based on the factor analyses performed in the original development of each instrument. In the pre-test analyses, the Cronbach $\alpha$ values fell between 0.70 and 0.90 . Thus, the final instrument was unaltered. In the final data collection, factor analyses were repeated and similar values of Cronbach's $\alpha$ were obtained, indicating that the scales at pre-test and in the final data collection were appropriate for this population.

\section{Data analysis}

Mean scores on each of the subscales and total scales were calculated for the women. Linear and logistic regression analyses were done controlling for demographic variables of the population to understand the impact of selected variables on the eating styles and body size preferences. Significance was set at $P$-values less than or equal to 0.05. Data were analysed using the Statistical Package for Social Sciences (SPSS) software for personal computers, release 13.0 (2005).

\section{Results}

\section{Demographics}

Table 1 presents the demographic distribution of the sample. The women were on average 33.5 years of age (standard deviation 11.271). The majority were married, Muslim, of medium-income families, medium socioeconomic status, with at least a high school degree and from urban settings. In addition, most women fell in the 
Table 1 Distribution of participants according to selected demographic variables

\begin{tabular}{|c|c|}
\hline Variable & Percentage $(n=800)$ \\
\hline \multicolumn{2}{|l|}{ Age (years) } \\
\hline $18-24$ & 23.7 \\
\hline $25-39$ & 48.6 \\
\hline $40-73$ & 27.7 \\
\hline \multicolumn{2}{|l|}{ Marital status } \\
\hline Single* & 31.9 \\
\hline Married & 68.1 \\
\hline \multicolumn{2}{|l|}{ Religion } \\
\hline Muslim & 96.8 \\
\hline Christian† & 3.2 \\
\hline \multicolumn{2}{|c|}{ Annual income (Jordanian Dinars) } \\
\hline$<250$ & 40.2 \\
\hline $250-499$ & 42.5 \\
\hline$>499$ & 17.3 \\
\hline \multicolumn{2}{|l|}{ Socio-economic status } \\
\hline Low & 12.0 \\
\hline Medium & 64.0 \\
\hline High & 24.0 \\
\hline \multicolumn{2}{|l|}{ Education level } \\
\hline Illiterate & 11.6 \\
\hline Less than high school & 18.4 \\
\hline High school & 37.2 \\
\hline More than high school & 32.1 \\
\hline \multicolumn{2}{|l|}{ Area of residence } \\
\hline Urban & 84.0 \\
\hline Rural & 16.0 \\
\hline \multicolumn{2}{|l|}{ Body mass index } \\
\hline Underweight & 3.4 \\
\hline Normal weight & 42.9 \\
\hline Overweight & 35.3 \\
\hline Obese & 18.5 \\
\hline
\end{tabular}

* Includes eight divorced, three separated and 28 widowed women. † Includes one 'Other'.

normal BMI category; however, the results also indicated high levels of overweight (35.3\%) and obesity (18.5\%).

\section{Hypotbesis 1}

The nutrition transition is underway in Jordan. Specifically, most Jordanian women will be restrained eaters.

Table 2 presents the mean scores (possible range 1-5) on each of physical, external and emotional eating. The women in this sample scored significantly higher on emotional eating (3.5992) compared with both physical and external eating (3.0880 and 3.2341, respectively). The emotional eating score was between 3 (neutral) and 4 (agree) (closer to 4), indicating that these women tended to agree that they eat based on emotional cues such as anger, frustration, joy and happiness. In addition, using the median of 10 as the cut-off point on the RS, $42.1 \%$ of the women were restrained eaters, thus indicating that a large number of these women restricted energy intake on a daily basis. Furthermore, using the cut-off point of 20 for disordered eating on the EAT-26 scale, $54.8 \%$ of the women had disordered eating attitudes and behaviours with a mean score of 18.99 .
Table 2 Mean scores on the different subscales and scales

\begin{tabular}{lcc}
\hline Scale/subscale & Mean & Standard deviation \\
\hline MFES & & \\
Physical & $3.0880^{*}$ & 0.7806 \\
$\quad$ External & $3.2341 \dagger$ & 0.6520 \\
$\quad$ Emotional & $3.5992^{*} \dagger$ & 0.8073 \\
EAT-26 & 18.9848 & 10.7603 \\
RS & 10.4115 & 5.6691 \\
SATAQ-3 & & \\
$\quad$ Information & 2.8725 & 0.9957 \\
$\quad$ Pressures & 2.1130 & 0.7066 \\
$\quad$ Internalisation-general & 2.6293 & 0.9762 \\
$\quad$ Internalisation-athlete & 2.8167 & 1.0077 \\
BES & & \\
$\quad$ BE-appearance & 2.6035 & 0.5889 \\
BE-weight & 2.8346 & 0.5701 \\
$\quad$ BE-attribution & 3.2090 & 0.8470 \\
BMI & 26.0875 & 5.2422 \\
Desired change & -1.7031 & 1.5782 \\
\hline
\end{tabular}

MFES - Motivation for Eating Scale; EAT-26 - Eating Attitudes Test; RS - Eating Habits Questionnaire, also known as the Revised Restraint Scale; SATAQ-3 - Sociocultural Attitudes Towards Appearance Scale; BES - Body Esteem Scale; BMI - body mass index.

* Mean is different from mean external eating at the 0.05 level of significance. $\dagger$ Mean is different from mean physical eating at the 0.05 level of significance.

On the other hand, women on average thought they were a 5 on the body silhouettes, which is consistent with their mean BMI of $26.1 \mathrm{~kg} \mathrm{~m}^{-2}$, and wanted to lose, on average, one body silhouette; they wanted to become a 4 , which is the healthiest body silhouette size. Only 5.4\% of the women wanted to be larger than a 5 , and only $8.5 \%$ wanted to be smaller than a 3 .

Furthermore, the mean scores for the BES subscales indicate that these women do not have substantial body esteem issues. For the three subscales, the mean scores fell between 2 (disagree) and 3 (neutral), and ranged from 2.60 to 3.21 ; indicating a rather neutral level of body esteem. Similarly, mean scores on the SATAQ-3 indicate that these women did not feel the media was dictating the way their body should look. The mean scores for three of the subscales (information, internalisation-general and internalisationathlete) were between 2 (disagree) and 3 (agree), and ranged from 2.63 and 2.87, while that of the pressures subscale (2.11) indicated a higher level of disagreement about feeling pressure from the media to look a certain way.

These results indicate that a significant percentage of Jordanian women in this sample are restrained, tend to agree that they eat based on emotional cues, and exhibit higher than expected levels of disordered eating attitudes and behaviours. While these women want to lose weight, they want to achieve a normal BMI rather than wanting to be ultra-thin. Furthermore, the results indicate neutral levels of body esteem issues and low impact of the media on body appearance in this sample.

\section{Hypotbesis 2}

Western advertising and media influences will explain the eating style and body size preferences of Jordanian women. 
Table 3 presents the unstandardised coefficients for all of the significant independent and mediating variables regressed on the scores for emotional eating, restrained eating, EAT-26 and desired body change. These four dependent variables were selected as an indication of where the women fell within the nutrition transition model. Each of the regression models included the three main independent variables: (1) area of residence, (2) BMI and (3) Western media and advertising (SATAQ-3). Control variables included religion, age, marital status, income, socio-economic status and education level. All models were linear regression models except for desired change which employed a logistic regression model, with the dependent variable being dichotomously split into wanting to lose weight versus wanting to stay the same or gain weight.

The results of the models were all in the expected direction. However, marital status, age, socio-economic status and religion were not significant in any of the models. These items were dropped from the final models and are not included in the table. This may indicate that for Jordanian women changes in the nutrition transition stage are more likely to be a reflection of cultural changes due to Western advertising and media than being due to age, socioeconomic status or religion. In addition, area of residence was significant only in the restrained eating model. Thus, those who lived in urban settings scored 2.391 more points on the RS, indicating higher levels of restrained eating.

Table 3 Unstandardised coefficients for independent variables regressed for emotional eating, restrained eating, EAT-26 and desired change

\begin{tabular}{|c|c|c|c|c|}
\hline Variable & Emotional & Restrained & EAT-26 & $\begin{array}{l}\text { Desired } \\
\text { change }\end{array}$ \\
\hline \multicolumn{5}{|l|}{ Urban } \\
\hline Yes & & $2.391^{*}$ & & \\
\hline No & & - & & \\
\hline \multicolumn{5}{|l|}{ SATAQ-3 } \\
\hline Information & & $1.679^{*}$ & & \\
\hline Pressures & $2.387^{*}$ & $5.909^{\star \star}$ & & $2.908^{\star \star}$ \\
\hline $\begin{array}{l}\text { Internalisation-general } \\
\text { Internalisation-athlete }\end{array}$ & $1.957^{\star}$ & $4.081^{* *}$ & $5.580^{\star *}$ & \\
\hline \multicolumn{5}{|l|}{ BMI } \\
\hline Underweight & & & & \\
\hline Normal & - & - & - & - \\
\hline Overweight & $2.077^{\star}$ & $6.543^{\star \star}$ & - & $11.136^{\star \star}$ \\
\hline Obese & $3.336^{* *}$ & $12.269^{\star \star}$ & $4.131^{\star \star}$ & $18.050^{\star \star}$ \\
\hline \multicolumn{5}{|l|}{ Income } \\
\hline Low & & - & - & \\
\hline Medium & & $2.033^{*}$ & $2.921^{\star \star}$ & \\
\hline High & & $3.574^{\star *}$ & $3.427^{\star *}$ & \\
\hline \multicolumn{5}{|l|}{ Education } \\
\hline Illiterate & - & & - & \\
\hline Less than high school & $4.885^{\star \star}$ & & $2.391^{*}$ & \\
\hline High school & $5.814^{\star *}$ & & $2.664^{\star *}$ & \\
\hline More than high school & $5.769^{\star \star}$ & & $3.640^{\star *}$ & \\
\hline Constant & $33.876^{\star \star}$ & $2.977^{\star \star}$ & $3.554^{\star \star}$ & \\
\hline$R^{2}$ adjusted/ - 2LL & 0.379 & 0.246 & 0.257 & 971.53 \\
\hline $\mathrm{N} / \mathrm{df}$ & 800 & 800 & 800 & 4 \\
\hline
\end{tabular}

EAT-26 - Eating Attitudes Test; SATAQ-3 - Sociocultural Attitudes Towards Appearance Scale; BMI - body mass index.

* Unstandardised coefficients were significant at the 0.05 level.

** Unstandardised coefficients were significant at the 0.01 level.
Exploring each of the models separately is revealing. First, the emotional eating model indicates that for every point scored on pressures from the media and internalising the general images, these women scored 2.387 and 1.957 points more on the emotional eating subscale indicating that if the women felt pressure from the media to look a certain way or if they internalised general media images, their emotional eating score was higher.

Furthermore, while being underweight compared with normal weight did not affect the emotional eating score, being overweight or obese, compared with being normal weight, meant an increase of 2.077 points and 3.336 points respectively on the emotional eating scale, indicating an eating style based more on emotional cues. Interestingly, increases in education also led to increases in the emotional eating score. This may be explained by the fact that emotional eating is usually linked to upper and middle classes that are more likely to be educated ${ }^{18}$. Thus, while socio-economic status was not significant here, it may have an indirect influence through education.

Second, the restrained eating model indicates that for every point scored on (1) pressures from the media, (2) internalising the general images and (3) internalising the athlete images, participating women scored 5.909, 4.081 and 3.345 points more on the restrained eating scale, indicating that they were more likely to restrict their energy intake. Furthermore, while being underweight, compared with being normal weight, did not affect the restrained eating scores, being overweight or obese compared with normal weight meant an increase of 6.543 points and 12.269 points, respectively, on the restrained eating scale. Furthermore, increases in income led to increases in the restrained eating score.

Third, the EAT-26 model indicates that for every point scored on media information regarding body image and internalising the general images in the media, participating women scored 1.679 and 5.580 points more on the EAT-26, indicating higher levels of disordered eating attitudes and behaviours. Similar to the other two models, being underweight compared with normal weight did not affect the EAT-26 score. However, being overweight also did not affect the EAT-26 score. Yet, being obese compared with normal weight meant an increase of 4.131 points on the EAT-26 score. Furthermore, increases in income and education both led to increases in the EAT-26 score.

Finally, the desired change model indicates, as expected, that those who felt more media pressure to look a certain way were 2.908 times more likely to want to lose weight compared with those who did not feel that pressure. Furthermore those who were overweight or obese were 11.136 and 18.050 times, respectively, more likely to want to lose weight compared with those who were normal weight. No other independent or control variables were found to be significant. Thus, the hypothesis that Western advertising and media explain the nutrition transition stage seems to be valid to at least a certain level. 


\section{Discussion}

Previous research indicates that the nutrition transition leads to shifts in eating styles and dietary patterns and thus to increases in the prevalence of obesity and NR-NCDs ${ }^{19}$. These effects can be attributed to increased urbanisation, improvements in socio-economic status and greater Western influences, especially Western advertising and media $^{19-22}$.

The results of this study confirm that the nutrition transition is well underway in Jordan. An anticipated high level of restrained eating was observed in the study population. Furthermore, the results indicate that alarming rates of disordered eating attitudes and behaviours exist in this population. Paradoxically, desired changes in body size were reasonable and healthy, and women's body esteem levels were relatively normal, given such high levels of restrained and emotional eating and the high prevalence of overweight and obesity found in this sample. This may be due to the fact that women are caught between a 'traditional' and a 'modern' society; a concept explored below.

As expected, women who were overweight or obese scored higher on emotional eating, restrained eating, disordered eating attitudes and behaviours, and were more likely to want to lose weight than those who were of normal weight. In addition, BMI remains an important issue to address, both as a consequence of and as a driving force for the nutrition transition underlying body esteem and body size preferences. Jordan, which is currently experiencing rapid increases in overweight and obesity, may also soon experience a dramatic increase in eating disorders and body esteem problems. Thus, there is a need to curb the obesity epidemic in this population which may be further fuelled through abnormal eating behaviours such as dieting and emotional eating.

Moreover, the results highlight some of the cultural complexities associated with Jordanian women's perceptions of themselves as their world becomes more Westernised in the most fundamental way - through food and health. Specifically, the desired change in body size for Jordanian women was smaller than seen in developed and developing countries ${ }^{23-26}$. While similar percentages of women (66\%) in this study compared with US women (60\%) reported body dissatisfaction ${ }^{27}$, the desired change was smaller. For example, in the USA, both Asian and white women identified the ideal body silhouette as 2.7, Hispanics as 2.9 and blacks as $3.1^{23}$. In this study Jordanian women identified their ideal body silhouette as 3.9, which is closest to the healthy-sized silhouette of 4 .

While many traditional societies prefer plumpness as a sign of feminine beauty and an indication of good health, high social status and fertility ${ }^{28}$, this was not the case in Jordan, where women actually wanted to have a 'normal' body size. Nevertheless, Jordan appears to follow the normal progression of the nutrition transition. Typically, the nutrition transition initially offers a welcome avenue for weight gain for women who may not have been able to increase their body size while consuming traditional $\operatorname{diets}^{29}$. Consequently, the nutrition transition leads to dramatic increases in the number of people who are overweight and obese $\mathrm{e}^{30}$. Thus, if the nutrition transition has been underway in Jordan for some time, then the high prevalence of overweight (35.3\%) and obesity (18.5\%) observed in this study may not be unexpected.

Even as the nutrition transition contributes to overweight and obesity, the growing presence of Western advertising and media generally promotes a 'culture of thinness' among some populations - especially younger affluent women ${ }^{31}$. In many societies, this paradox may contribute to escalating rates of body dissatisfaction ${ }^{32}$, unhealthy dieting behaviours ${ }^{33}$ and the development of eating disorders ${ }^{34,35}$. In the case of Jordan, unhealthy dieting behaviours and disordered eating attitudes and behaviours were observed, but body dissatisfaction was not a problem. Thus, women in Jordan may be caught in a classic 'catch 22' where dietary patterns and eating habits are becoming more 'modern', while body esteem and body size preferences remain more 'traditional'. Additionally, these women may be internalising concepts and behaviours such as 'dieting' without really understanding why they are adopting these behaviours. Thus, women seem to be caught between two worlds; a traditional one that dictates cultural aspects of beauty and a modern one that bombards them with a larger variety of food options and allows them to access it in larger quantities. It also remains unclear from the data whether this concept of 'catch 22' is a temporary response to the nutrition transition. It remains to be seen whether younger women in this population will maintain this response to the nutrition transition, or whether Western advertising and media will push these women to respond in a more anticipated manner where body size preferences shift towards an ultra-thin ideal. Studying similar issues in a sample of young adolescent females may help better understand the extent of the problem, as younger adolescent females in most other cultural settings studied had a preference for thinner bodies and had lower body esteem than older females ${ }^{25,26,36}$.

\section{Implications and future research}

First, as indicated above, there is a need to understand the impact of Western advertising and media in a younger sample of this population. Second, the information identified regarding dieting behaviours may be useful in developing tailored education programmes with a focus on overweight and obesity and methods of controlling and preventing it in this population. From this study, it is clear that Jordan is currently at a most opportune time to prevent body image issues from arising among these women. Public health efforts should build on the current 
cultural preferences of healthy body size and develop school health curricula that emphasise body esteem at a young age and help deconstruct negative media messages regarding body size. Third, research measuring the levels of eating disorders is warranted. High levels of disordered eating attitudes and behaviours observed here may be indicative of high levels of eating disorders. While accurate measurements for eating disorders are not easy, there is a critical need to understand the magnitude of eating disorders in this population.

There are two limitations of the current study: first, this research was not representative at the governorate level; second, measuring the impact of Western advertising and media may need to be further tested and expanded. However, critical baseline information was provided through this study.

\section{Conclusions}

This study lends support for Hawks et al.'s nutrition transition model ${ }^{4}$. In addition, it shows that Western advertising and media may have more subconscious influences on women's eating styles than anticipated. It also verifies the consequences of rising levels of overweight and obesity in a population especially with regard to emotional and restrained eating, disordered eating attitudes and behaviours.

\section{Acknowledgements}

Sources offunding: This research was funded by the David M Kennedy Center for International Studies and the Family Studies Center at Brigham Young University. The authors would also like to thank the Department of Statistics in Jordan for its collaboration.

Conflict of interest declaration: None declared.

Authorship responsibilities: H.N.M. designed and carried out the research, and wrote the manuscript. R.B.B. and S.R.H. supervised the research and revised the manuscript.

\section{References}

1 Drewnowski A. Nutrition transition and global dietary trends. Nutrition 2000; 16(7-8): 486-7.

2 Popkin BM. An overview on the nutrition transition and its health implications: the Bellagio meeting. Public Health Nutrition 2002; 5(1A): 93-103.

3 Popkin BM. The shift in stages of the nutrition transition in the developing world differs from past experiences. Public Health Nutrition 2002; 5(1A): 205-14.

4 Hawks SR, Merrill RM, Madanat HN, Miyagawa T, Suwanteerangkul J, Guarin CM, et al. Intuitive eating and the nutrition transition in Asia. Asia Pacific Journal of Clinical Nutrition 2004; 13(2): 194-203.

5 Takruri H. FAO-Nutrition Country Profile: Jordan. Rome: Food and Agriculture Organization, 2003.

6 Bloom DE, Canning D, Huzarski K, Levy D, Nandakumar AK, Sevilla J, et al. Demographic Transition and Economic Opportunity: the Case of Jordan [online] 2001.
Available at http://www.phrplus.org/Pubs/Tech011_fin. pdf/. Accessed January 2007.

7 Centers for Disease Control and Prevention. Prevalence of selected risk factors for chronic disease - Jordan, 2002. Morbidity and Mortality Weekly Report 2003; 52(43): 1042-4.

8 Demographic and Health Survey for Jordan. Nutritional Status, Prevalence of Anemia, and Micronutrient Supplementation [online], 2002. Available at http://www. measuredhs.com/pubs/pdf/FR138/10Chapter10.pdf. Accessed January 2007.

9 Hawks SR, Madanat HN, Merrill RM, Goudy MB, Miyagawa T. A cross-cultural analysis of 'motivation for eating' as a potential factor in the emergence of global obesity: Japan and the United States. Health Promotion International 2003; 18(2): $153-62$.

10 Drewnowski A, Riskey D, Desor JA. Measures of restraint: separating dieting from overweight. Appetite 1982; 3(3): 282.

11 Polivy J, Herman PC, Warsh S. Internal and external component of emotionality in restrained and unrestrained eaters. Journal of Abnormal Psychology 1978; 87(5): 497-504.

12 al-Subaie A, al-Shammari S, Bamgboye E, al-Sabhan K, al-Shehri S, Bannah AR. Validity of the Arabic version of the Eating Attitude Test. International Journal of Eating Disorders 1996; 20(3): 321-4.

13 Garner DM, Garfinkel PE. The Eating Attitudes Test: an index of the symptoms of anorexia nervosa. Psychological Medicine 1979; 9(2): 273-9.

14 Lorenzo CV, Lavori PW, Lock JD. Eating attitudes in high school students in the Philippines: a preliminary study. Eating and Weight Disorders 2002; 7(3): 202-9.

15 Thompson JK, van den Berg P, Roehrig M, Guarda AS, Heinberg LJ. The sociocultural attitudes towards appearance scale-3 (SATAQ-3): development and validation. International Journal of Eating Disorders 2004; 35(3): 293-304.

16 Stunkard AJ, Sorensen T, Schulsinger F. Use of the Danish adoption register for the study of obesity and thinness. In: The Genetics of Neurological and Psychiatric Disorders. New York: Raven Press, 1983; 115-20.

17 Mendelson BK, Mendelson MJ, White DR. Body-esteem scale for adolescents and adults. Journal of Personality Assessment 2001; 76(1): 90-106.

18 Rasheed P. Perception of body weight and self-reported eating and exercise behaviour among obese and non-obese women in Saudi Arabia. Public Health 1998; 112(6): 409-14.

19 Popkin BM, Gordon-Larsen P. The nutrition transition: worldwide obesity dynamics and their determinants. International Journal of Obesity and Related Metabolic Disorders 2004; 28(Suppl. 3): S2-9.

20 Adair LS. Dramatic rise in overweight and obesity in adult Filipino women and risk of hypertension. Obesity Research 2004; 12(8): 1335-41.

21 Kennedy ET. The global face of nutrition: what can governments and industry do? Journal of Nutrition 2005; 135(4): 913-5.

22 Haslam DW, James WP. Obesity. Lancet 2005; 366(9492): 1197-209.

23 Cachelin FM, Rebeck RM, Chung GH, Pelayo E. Does ethnicity influence body-size preference? A comparison of body image and body size. Obesity Research 2002; 10(3): 158-66.

24 Contento IR, Basch C, Zybert P. Body image, weight, and food choices of Latina women and their young children. Journal of Nutrition Education and Behavior 2003; 35(5): 236-48.

25 Jackson RT, Rashed M, Saad-Eldin R. Rural-urban differences in weight, body image, and dieting behavior among adolescent Egyptian schoolgirls. International Journal of Food Sciences and Nutrition 2003; 54(1): 1-11. 
26 McIza Z, Goedecke JH, Steyn NP, Charlton K, Puoane T, Meltzer S, et al. Development and validation of instruments measuring body image and body weight dissatisfaction in South African mothers and their daughters. Public Health Nutrition 2005; 8(5): 509-19.

27 Mossavar-Rahmani Y, Pelto GH, Ferris AM, Allen LH. Determinants of body size perceptions and dieting behavior in a multiethnic group of hospital staff women. Journal of the American Dietetic Association 1996; 96(3): 252-6.

28 Treloar C, Porteous J, Hassan F, Kasniyah N, Lakshmanudu $\mathrm{M}$, Sama M, et al. The cross cultural context of obesity: an INCLEN multicenter collaborative study. Health \& Place 1999; 5(4): 279-86.

29 Holdsworth M, Gartner A, Landais E, Maire B, Delpeuch F. Perceptions of healthy and desirable body size in urban Senegalese women. International Journal of Obesity and Related Metabolic Disorders 2004; 28(12): 1561-8.

30 Popkin BM, Horton S, Kim S, Mahal A, Shuigao J. Trends in diet, nutritional status, and diet-related noncommunicable diseases in China and India: the economic costs of the nutrition transition. Nutrition Reviews 2001; 59(12): 379-90.
31 Nasser M. Culture and weight consciousness. Journal of Psychosomatic Research 1988; 32(6): 573-7.

32 Groesz LM, Levine MP, Murnen SK. The effect of experimental presentation of thin media images on body satisfaction: a meta-analytic review. International Journal of Eating Disorders 2002; 31(1): 1-16.

33 Gunewardene A, Huon GF, Zheng R. Exposure to Westernization and dieting: a cross-cultural study. International Journal of Eating Disorders 2001; 29(3): 289-93.

34 Lake AJ, Staiger PK, Glowinski H. Effect of Western culture on women's attitudes to eating and perceptions of body shape. International Journal of Eating Disorders 2000; 27(1): 83-9.

35 Makino M, Tsuboi K, Dennerstein L. Prevalence of eating disorders: a comparison of Western and non-Western countries. Medscape General Medicine 2004; 6(3): 49.

36 Rand CS, Wright BA. Thinner females and heavier males: who says? A comparison of female to male ideal body sizes across a wide age span. International Journal of Eating Disorders 2001; 29(1): 45-50. 